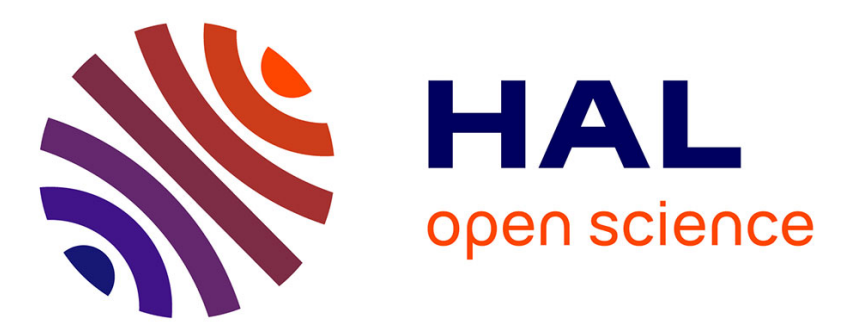

\title{
Modélisation des mouvements articulatoires par la méthode de la LPC multi-impulsionnelle
}

\author{
S. Bouabana, S. Maeda
}

\section{To cite this version:}

S. Bouabana, S. Maeda. Modélisation des mouvements articulatoires par la méthode de la LPC multi-impulsionnelle. Journal de Physique IV Proceedings, 1994, 04 (C5), pp.C5-449-C5-452. 10.1051/jp4:1994593 . jpa-00253088

\section{HAL Id: jpa-00253088 https://hal.science/jpa-00253088}

Submitted on 1 Jan 1994

HAL is a multi-disciplinary open access archive for the deposit and dissemination of scientific research documents, whether they are published or not. The documents may come from teaching and research institutions in France or abroad, or from public or private research centers.
L'archive ouverte pluridisciplinaire HAL, est destinée au dépôt et à la diffusion de documents scientifiques de niveau recherche, publiés ou non, émanant des établissements d'enseignement et de recherche français ou étrangers, des laboratoires publics ou privés. 


\title{
Modélisation des mouvements articulatoires par la méthode de la LPC multi-impulsionnelle
}

\author{
S. BOUABANA et S. MAEDA
}

ENST, Télécom Paris, Département Signal, URA 828 du CNRS, 46 nue Barrault, 75634 Paris cedex 13, France

\begin{abstract}
Are articulatory commands organized in terms of phonemes, syllables or others phonetic units? We attempt to examine this question by modeling articulatory movements with the help of an LPC method where the excitation source is represented by a multi-pulse sequence. Our results deviate from the traditional phonemic-concatenation theory and favor that the elementary gesture in speech production corresponds to the syllabic gesture.
\end{abstract}

\section{Introduction}

La parole est communément vue comme le résultat de la concaténation de segments phonétiques. Cependant, la question sur la nature de ces segments reste toujours posée. Les premières tentatives de synthèse segmentale à partir de phonèmes ont abouti à l'échec. Les transitions entre phonèmes nécessaires pour l'intelligibilité de la parole ont, en effet, été omises. Pour pallier à ce problème, des informations supplémentaires (lexicales, syntactiques, sémantiques..) sous forme de règles ont été utilisées pour la conversion phonèmes/parole [1]. Un autre moyen moins lourd mais plus rigide, consiste à utiliser le diphone (segment de parole entre les instants de stabilité maximale de deux phonèmes) comme unité de concaténation [2]. La variabilité contextuelle de certains phonèmes constitue une sérieuse entrave au raccordement des diphones. Certains systèmes ont donc recours à des ensembles d'unités plus étendus : di-syllabes, pour minimiser les difficultés de concaténation [3]. Cette solution est, cependant, très encombrante. Un groupe de chercheurs français a proposé le polyson comme une sorte de compromis entre les diphones et les di-syllabes [4]. Le polyson est défini comme une unité segmentale aux frontières de laquelle ne se situent que des phonèmes spectralement stables et peu sujet à la variabilité.

L'objectif de cet article, est de contribuer à la discussion sur l'unité phonétique en partant de la modélisation des mouvements articulatoires du conduit vocal par la méthode de la LPC multiimpulsionnelle. Notre étude porte sur l'analyse du mouvement de la langue durant la production de la parole. Cet articulateur, qui constitue la partie la plus mobile du système supraglottique de l'appareil vocal, participe très activement à la production. Maeda [5] a décrit les variations temporelles du profil de la langue à l'aide de 4 composantes linéaires extraites de l'analyse factorielle appliquée à des données radiofilms [6] : une composante extrinsèque, la position de la mâchoire (fermé/ouvert); trois composantes intrinsèques, la position du corps de la langue (antérieur/postérieur), la forme du corps de la langue (arquée/plate) et la position de l'apex de la langue (haute/basse). Ces composantes décrivent environ $90 \%$ de la variance du profil de la langue observée durant la parole.

Nous disposons au total de 10 phrases françaises prononcées par deux locuteurs, PB et DF, et enregistrées à une fréquence de 50 images $/ \mathrm{sec}$. La figure 1 illustre un exemple de l'évolution temporelle du paramètre "position de la langue " pour la phrase " Louis pense à ça "(courbe en trait plein). 


\section{Position du problème}

On suppose qu'on peut décrire la variation temporelle de chacun des paramètres articulatoires (mouvement) séparément, par un modèle source-filtre. Pour chaque modèle, la source est décrite par un train d'impulsions et le filtre est supposé de type auto-régressif (AR). Le problème est, alors, de séparer la commande musculaire (source) de la réponse impulsionnelle (filtre) à partir des signaux d'observations. Une solution consiste à identifier le modèle du filtre auto-régressif et ensuite, à déterminer l'excitation en deux étapes.

\section{Identification du modèle}

La modélisation AR a été précédée par un traitement particulier appelé pré-accentuation. Cette opération est obtenue par un filtre différentiateur du premier ordre qui dépend d'un coefficient $\alpha$. En phase d'analyse, son rôle est d'applatir la pente spectrale du signal d'observation, afin de faciliter la détection de pôles. Le coefficient $\alpha$ a été déterminé empiriquement tel que l'énergie de l'erreur entre le signal synthétisé et le signal observé soit faible. Cette valeur est donnée par $\alpha=0.5$. En synthèse, l'effet de ce filtre est traduit par un filtre de dé-accentuation ou filtre intégrateur de même coefficient. Ce filtre permet la mise en forme du signal d'excitation.

Dans un problème d'identification du modèle du filtre, on est d'abord confronté à la détermination de l'ordre du modèle $p$. Dans le cas d'un modèle AR, Akaîke a proposé un critère pour déterminer l'ordre du filtre [7]. Le critère $A I C(p)$ ou Akaïke Information Criterion est défini comme $A I C(p) \triangleq$ $\log \left(\nabla_{p}^{*} / R_{0}\right)+2 p / k N . \quad N, \nabla_{p}^{*}, R_{0}$ et $k$ sont respectivement le nombre des échantillons du signal, l'énergie de l'erreur de prédiction pour $p$ pôles, la variance du signal et le coefficient de fenêtrage ( $k=0.375$ dans le cas où les signaux sont filtrés par un filtre de Hanning). La valeur $p$ est donnée par le minimum de $A I C(p)$. Le tracé de la fonction $A I C(p)$, pour les quatre paramètres sur l'ensemble des dix phrases, nous a conduit à choisir $p=2$. Cette valeur est obtenue dans $90 \%$ des cas.

En résumé, le modèle de synthèse se compose de deux parties : un filtre de dé-accentuation d'ordre 1 et un filtre AR d'ordre 2 à identifier.

Une fois l'ordre du filtre déterminé, l'identification des coefficients $a_{i}, i=1, \ldots, p$ est effectuée par la technique de la LPC. Cette méthode consiste à minimiser l'énergie de l'erreur entre le signal réel $y(n)$ et le signal synthétisé $\hat{y}(n)$. La résolution de cette optimisation par la méthode de corrélation fournit aisément les coefficients du modèle.

L'identification des coefficients du filtre AR pour chaque paramètre articulatoire a été effectuée pour l'ensemble des phrases données. Ceci nous a permis de calculer les paires de pôles conjugués correspondant que nous avons reportés sur le cercle unité. Pour chaque paramètre, deux nuages de points compacts apparaîssent de part et d'autre de l'axe réel positif. Pour avoir des systèmes représentatifs de l'invariance temporelle, les quatre filtres de synthèse sont construits à partir des centres de gravité des nuages de points respectifs. Les réponses impulsionnelles de ces quatre filtres AR correspondent à des réponses stables présentant une faible oscillation. Comme le signal de commande est une séquence d'impulsions, ces réponses impulsionnelles sont considérées comme le geste élémentaire qui, par superposition, donnent naissance aux mouvements articulatoires. Il est important de remarquer que la durée effective de chacune des réponses impulsionnelles est comprise entre 140 et $200 \mathrm{~ms}$. Cette durée est pratiquement égale à la durée moyenne de la syllabe française $180 \mathrm{~ms}$.

\section{Détermination de l'excitation}

Supposons que $\hat{y}_{m}(n)$ est la sortie du synthétiseur AR excité par une séquence $v_{m}(n)$ qui n'est pas nulle pour $m$ instants fini. L'estimation de $v_{m}(n)$ est choisie de manière à minimiser $\sum_{n=1}^{N}\left(\left|y(n)-\hat{y}_{m}(n)\right|^{2}\right)$. Une solution sub-optimale mais efficace est itérative sur $m$. Elle utilise $e_{m-1}(n) \triangleq y(n)-\hat{y}_{m-1}(n)$ 
pour déterminer la $m^{\text {eme }}$ position. L'amplitude des impulsions déjà déterminées est recalculée à chaque nouvelle itération [8].

La qualité du mouvement synthétisé en fonction du nombre d'impulsions de la commande d'entrée est illustrée par la figure 1. Cette figure montre que la différence entre les deux mouvements observé et reconstitué diminue lorsque le nombre des impulsions augmente. Afin d'illucider ce résultat, nous avons calculé l'énergie de l'erreur entre les deux signaux en fonction du nombre d'impulsions $m$, comme $E(m) \triangleq\left[\sum_{n=1}^{N}\left|e_{m}(n)\right|^{2}\right] /\left[\sum_{n=1}^{N} y(n)^{2}\right]$. Nous avons observé, ainsi que le montre l'exemple de la figure 2, que l'erreur diminue rapidement pour un nombre d'impulsions égal au nombre de syllabes dans la phrase, en l'occurence $m=4$. Si bien que la pente de cette portion de la courbe coupe l'axe des abscisses au point $m$ égal à 4 . Au delà de cette valeur et jusqu'à $m$ égal au nombre de phonèmes, $m=10$, la pente de l'erreur devient relativement faible. Dans cette partie du graphe l'énergie de l'erreur est inférieure à $10 \%$ de l'énergie du signal observé. Une saturation est observée, lorsque $m$ est supérieur au nombre de phonèmes. Remarquons que pour quelques exemples ces résultats ne sont pas rigoureusement vérifiés.

\section{Discussions et conclusion}

Dans le paragraphe 3 nous avons décrit le modèle de synthèse à l'aide de deux filtres. Dans la chaine de production de la parole chez l'homme, on distingue deux systèmes : un système physiologique et un système dynamique (mécanique). Le problème est d'établir une analogie entre notre modèle et la chaîne de production. Nos premières recherches nous ont conduits à choisir le filtre AR (ordre 2) comme système dynamique. Le mouvement articulatoire est, en effet, souvent considéré comme un mouvement dynamique caractérisé par une masse $M$, un facteur d'amortissement $B$ et une constante de raideur $K$ décrit par l'équation de Newton $M \ddot{y}(t)+B \dot{y}(t)+K y(t)=f(t)$, où $f(t)$ est la force d'excitation $[9,10]$. Le filtre de dé-accentuation correspond, alors, au système physiologique. Il consiste à transformer la séquence d'impulsions en une forme d'onde exponentielle qui repésente la force excitatrice du système dynamique, $f(t)$. Toutefois, pour modéliser le mouvement de la main durant l'écriture, Yasuhara a identifié deux systèmes [11]. Il a associé le système composé par la paire de pôles complexes conjugués au système physiologique, et le système fortement amorti par l'effet du pôle réel (le deuxième pôle étant pratiquement nul) au mouvement dynamique de la main. Dans ce cas, notre filtre de prétraitement d'ordre 1 correspond au système mécanique; il est donc visco-élastique.

Dans notre travail, nous avons modélisé les paramètres articulatoires de la langue par de simples filtres LPC excités par un nombre fini d'impulsions. La détection et l'estimation des impulsions sont basées sur la procédure de l'analyse-par-synthèse. L'interprétation phonétique des résultats obtenus suggère que l'organisation articulatoire au niveau neuro-musculaire entraîne la production d'un mouvement articulatoire élémentaire dont la durée est comparable à celle de la syllabe.

Ce travail entre dans le cadre du projet européen Esprit/BRA, no6975, SP, SPEECHMAPS.

\section{References}

[1] Klatt D.H., Review of text-to-speech conversion for English. JASA, 82(3) (1987) pp. 737-793.

[2] Moulines E., Charpentier F., Pitch-synchronous waveform processing techniques for text-to-speech synthesis using diphones. Speech Comm., 9 (1990) pp.453-467

[3] Fujimura O., Phonology and phonetics : A syllable-based model of articulatory organization. JASJ, (English Series) 13(1) (1992) pp. 39-48.

[4] Laferrière P., Chollet G., Miclet L. et Tubach J.P., Segmentation d'une base de données de "polysons", application à la synthèse de la parole. $X I V^{\text {eme }} \mathrm{JEP}, 6$ (1985) pp. 107-110.

[5] Maeda, S. Modélisation articulatoire du conduit vocal. Journal de Physique IV(2) (1992) pp.307-314.

[6] Bothorel A., Simon P., Wioland F. et Zerling J.P., Cinéradiographie des voyelles et consonnes du Français. Travaux de l'Institut de Phonétique, (1986).

[7] Makhoul J., Linear prediction : A tutorial review. Proc. IEEE, 63(4) (1975).

[8] Atal B.S. et Remde R., High-quality speech at low bit rates : Multi-pulse and stochastically excited linear predictive coders. Proc. ICASSP, (1986) pp. 614-617. 
[9] Browman C.P. et McGowan R.S., Extracting dynamic parameters from speech movement data. JASA, 93(3) (1992) pp. 1580-1588.

[10] Sonoda Y. et Kiritani S., Analysis of tongue point movements by linear second-order system model. Ann. Bull. RILP, 10, (1976) pp. 29-35.

(11) Yasuhara M., Identification and Decomposition of fast handwriting system. IEEE Transaction on Circuits and Systems, 30(11) (1983) pp. 828-832.
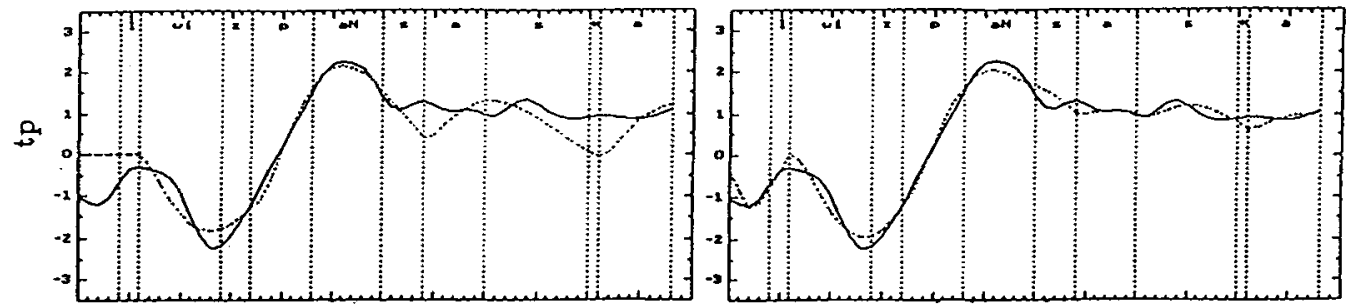

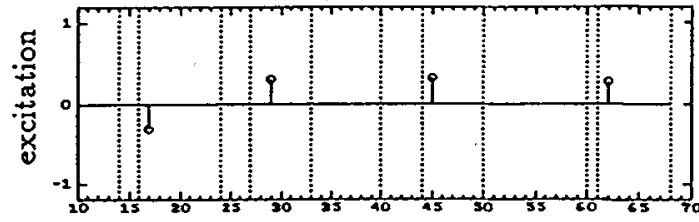

Nombre de trames $(20 \mathrm{~ms} /$ trame $)$

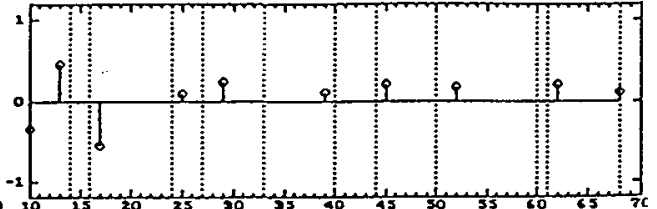

Nombre de trames (20ms/trame)

Figure 1: Evolution temporelle du paramètre "position de la langue" observé (trait plein) et synthétisé (pointillé). Nombre d'impulsions : $m=4$ (nombre de syllabes) et $m=10$ (nombre de phonèmes). Sujet $=\mathrm{PB}$, phrase $=$ "Louise pense à ça", $\alpha=0.5$
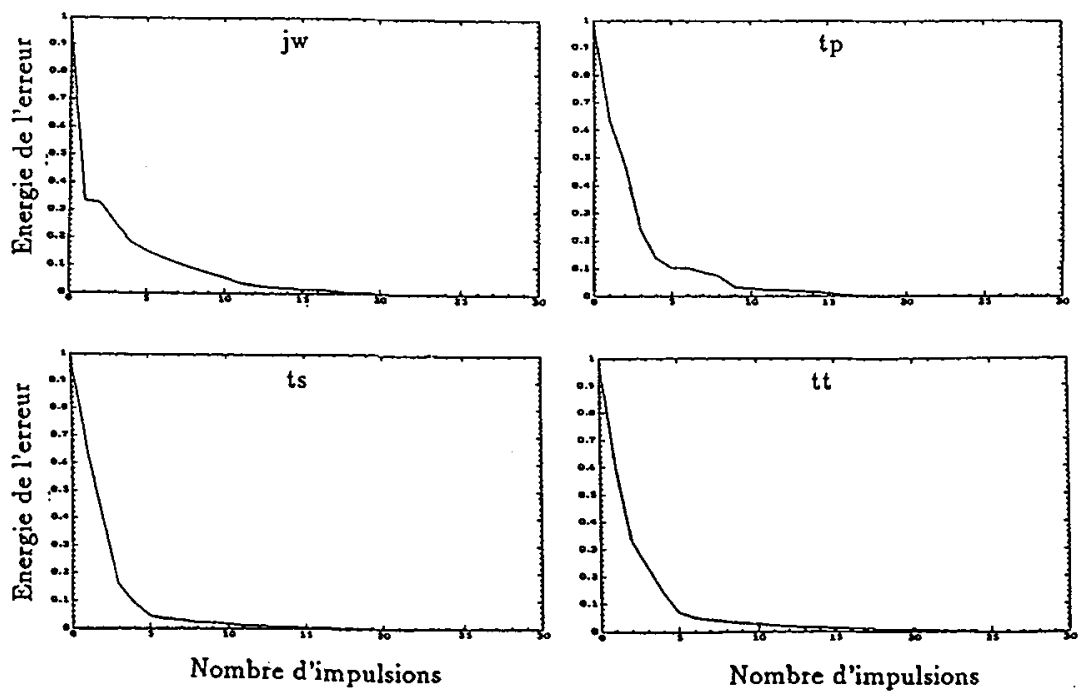

Figure 2: Energie de l'erreur en fonction du nombre d'impulsions pour les 4 paramètres articulatoires. 